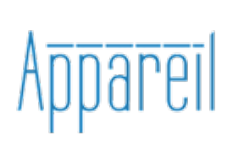

Appareil

$22 \mid 2020$

Une philosophie de l'appareil

\title{
Le nom, la carte et le monde
}

Essai en hommage à Jean-Louis Déotte

Román Domínguez Jiménez

\section{(2) OpenEdition}

Journals

Édition électronique

URL : http://journals.openedition.org/appareil/3462

DOI : 10.4000/appareil.3462

ISSN : 2101-0714

Éditeur

MSH Paris Nord

Référence électronique

Román Domínguez Jiménez, "Le nom, la carte et le monde », Appareil [En ligne], 22 | 2020, mis en ligne le 21 décembre 2020, consulté le 26 mars 2021. URL : http://journals.openedition.org/appareil/ 3462 ; DOI : https://doi.org/10.4000/appareil.3462

Ce document a été généré automatiquement le 26 mars 2021.

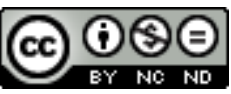

Appareil est mis à disposition selon les termes de la Licence Creative Commons Attribution - Pas d'Utilisation Commerciale - Pas de Modification 4.0 International. 


\title{
Le nom, la carte et le monde
}

\author{
Essai en hommage à Jean-Louis Déotte
}

Román Domínguez Jiménez

\section{NOTE DE L'ÉDITEUR}

Ce texte reprend des arguments et emprunte certains passages à un texte que l'auteur a rédigé à peu près en même temps en espagnol : « Del mundo como acontecimiento técnico y estético: una respuesta póstuma a Jean-Louis Déotte (1946-2018) », qui sera publié en Argentine en 2020 dans un recueil sur la philosophie de la technique.

\section{NOTE DE L'AUTEUR}

J'ai rédigé ce texte comme une réponse tardive à la dernière question que Déotte m'a posé dans notre dernier entretien. J'aurais voulu que Jean-Louis ait eût l'occasion de lire ma proposition, même si ce ne fût que pour la réfuter gentiment.

For the name of a man is a numbing blow from which he never recovers. Marshall McLuhan

On ne s'étonnera donc pas de rapprocher le faireépoque et l'invention - ou la nomination -, car celui qui nomme, c'est par excellence Adam, celui qui vient après la Création et retrouve le nom divin. Inventer, c'est donc nommer pour la seconde fois, renommer la Création divine.

Jean-Louis Déotte

En los desiertos del Oeste perduran despedazadas Ruinas del Mapa, habitadas por Animales y por Mendigos; en todo el País no hay otra reliquia de 
las Disciplinas Geográficas.

Jorge Luis Borges

J'écris ton nom.

Paul Éluard

\section{Le nom propre, un fil souterrain de l'œuvre de Déotte}

1 Lors de l'invitation que Audrey Rieber m'a très gentiment adressé pour participer, au moyen d'un texte, à cet hommage à Jean-Louis Déotte, il m'est venu à l'esprit de rédiger mon texte comme une sorte de réponse posthume au dernier entretien que j'ai eu avec Jean-Louis. En effet, à peu près deux semaines avant sa disparition, le 2 février 2018, Jean-Louis m'a adressé un courrier électronique, où, parmi d'autres soucis, il me posait la question: "et toi, quelles sont tes recherches?». Jean-Louis et moi, nous étions habitués à prendre un peu de temps, tout comme les gens étaient habitués à le faire au temps de la correspondance papier, pour répondre à nos messages. J'aurais aimé que Jean-Louis eût pu me donner son avis sur une intuition qui me hantait depuis longtemps, mais qui avait pris forme seulement dans les mois ayant précédé la cruelle disparition de mon maitre, professeur, compagnon et ami. Avant de déployer cette intuition, il faut cependant exposer les conditions qui m'ont amené à elle : vers la fin 2017, suite à la relecture de son texte sur le célèbre Laocoon de Gotthold Ephraim Lessing ${ }^{1}$, que je voulais utiliser dans mon cours d'esthétique moderne à l'institut d'Esthétique de l'université catholique de Chili, j'ai écrit à Jean-Louis pour savoir quelle était la source de son inspiration, outre l'évidente influence de la Namensprache de Benjamin ${ }^{2}$, ou à partir de quel auteur il avait établi sa pensée sur le nom propre. Mon hypothèse, c'est qu'un des fils souterrains de l'œuvre de Déotte serait justement la question du nom propre : le nom serait une sorte de trace indélébile qui transforme les corps, qui traverse comme un songe muet les domaines des appareils et des normes d'écriture, voire le passage entre des cosmétiques. Le souci des noms est présent chez Déotte dès son mémoire de DEA Un roi pour Israël ?3, jusqu'à son ouvrage posthume Cosmétiques ${ }^{4}$ et même au Mémorial de Caen, dont il a été le concepteur de la Fresque de noms. Toutefois, c'est lors de la lecture de Déotte sur la distinction que Lessing établit entre l'art plastique (la peinture) et la littérature (la poésie) que la question du nom propre émerge, du moins à mes yeux, de la manière la plus limpide dans son travail. Déotte observe que, pour Lessing, tandis que la peinture serait un art « de ce qui arrive aux corps, de leur réceptivité, de la passibilité des Corps. De ce qui marque le corps ${ }^{5}$ », c'est-à-dire de l'évènement et donc, du suspens, la poésie serait « au contraire un art de l'action, de l'action de ce qui transmet ou de qui se transmet au présent. Elle privilégie les traces d'un passage ${ }^{6} »$, c'est-à-dire de ce qui n'est pas évènement, donc, de ce qui subsiste dans la durée et malgré les mutations. D'un côté on a la couleur, l'espace, «l'instant fécond ${ }^{7}$ » qui marque le corps ; de l'autre, le temps, les métamorphoses, le fil sans lequel les actions ne sauraient devenir des traits

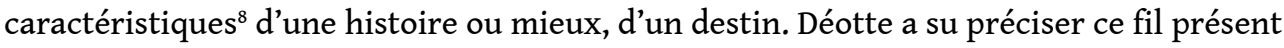
dans le texte de Lessing comme étant un nom : «ce qui subsiste peut n'être dit que par un nom. Le nom est bien ce qui transmet ce qui se transmet. Le nom désigne les traces d'un passage. Mieux, le nom peut concentrer les traces de l'action en étant lui-même ce qui agit ${ }^{9}$ ». C'est cette caractérisation du nom que Déotte trace à partir de Lessing qui m'a étonné, parce que, bien évidemment, le texte de Lessing utilise des noms propres, mais seule une lecture attentive comme celle de Déotte aurait pu extraire, et je dirai, 
presque deviner, qu'en effet la question de la poésie, en opposition aux marques évènementielles sur les corps subissants de la plastique, n'est rien d'autre que la marque incorporelle des noms propres. Pas de poésie donc, ou pas de littérature si l'on veut, qui n'appelle pas un nom, c'est-à-dire une marque singulière du corps-action dans la durée : « le nom de qui subsiste dans la métamorphose d'images ${ }^{10}$ ».

2 Alors, bien que j'aie eu pendant quelque temps l'intuition que la question du nom propre, tout en étant en quelque mesure négligée dans les études esthétiques, pourrait devenir un sujet important pour mes recherches à l'avenir, ce n'est qu'à partir du développement de la question élaboré par Déotte que j'ai pu engager une recherche concrète autour du nom propre Amérique. Au demeurant, je ne dis aucunement signification du nom, parce qu'en suivant la source de Déotte, qui est Le Différend de JeanFrançois Lyotard, qui à son tour s'inspire de Naming and Necessity de Saul Kripke ${ }^{11}$, un nom propre n'a pas de signification : «On peut valider les propriétés attribuées à l'être humain désigné par ce nom, mais pas son nom. Celui-ci n'ajoute aucune propriété. Même si beaucoup de noms ont initialement une signification, ils la perdent, et doivent la perdre ${ }^{12} »$. Le nom n'est qu'un index, un quasi-déictique ou un déictique hérétique, ensorcelant, en ceci que tandis que le déictique désigne un objet, un sujet ou une relation qui dépend de la situation et du contexte, le nom propre, bien qu'il soit, tout comme le déictique, un désignateur ou un index sans signification, reste toujours le même dans les phrases et les actions, comme une sorte de charme ou de sortilège que la parole exerce sur une entité, sur une réalité, sur un corps. Alors, pour moi, la question de l'archéologie de la désignation de l'Amérique ou des Amériques, ne saurait plus passer par ses significations, ses amphibologies et ses différents usages sémantiques, mais par une dispute sans fin, un différend autour du nom propre Amérique ou, pour reprendre le titre d'un ouvrage de l'historien chilien Miguel Rojas Mix, des Cent noms d'Amérique $^{13}$. Ma recherche prit la forme de deux textes qui traitent la question du nom Amérique $^{14}$ : au contraire des noms des autres continents, le nom Amérique serait le porteur d'une sorte de mythe ou plutôt d'une fantasmagorie moderne, et, en tant que moderne, un nom qui porterait l'empreinte de l'appareil de la perspective, car Amérique est née comme une désignation cartographique (d'ailleurs seulement de ce qui est aujourd'hui le Brésil) dans la carte mondiale de Martin Waldseemüller de 1507. C'est qu'au contraire des cartes médiévales (tels les portulans), les cartes modernes impliquent l'opération de la projection. Mon hypothèse, c'est que l'Amérique serait surtout le nom d'une anomalie en quelque sorte anachronique, le nom d'une survivance du mythe (Déotte lui-même rapporte le mythe à la norme d'écriture narrative des sociétés païennes ou dites primitives ${ }^{15}$ ) dans l'écriture projective: un mythe qui substitue la durée sans relâche vers l'avenir du progrès à la durée cyclique des mythes anciens et des religions révélées ${ }^{16}$.

3 Si l'Amérique en tant que miroir de l'Europe porte en elle-même l'empreinte du progrès, on peut esquisser l'hypothèse qu'Amérique serait l'un des noms de la modernité occidentale. Mieux: Amérique aurait été le nom ou la toponymie fantasmatique du progrès sur la carte mondiale, un nom projectif qui aurait eu un parcours qui va des cartes projectives aux romans, aux essais, manifestes et tracts des Américains des deux Amériques (car, du moins pour les Latino-américains, l'Amérique ne se limite pas du tout aux États-Unis), jusqu'au cinéma. Le cinéma serait l'appareil ayant su le mieux s'approprier la puissance mythique et fantasmagorique de ce nom: nom du progrès et des vastes territoires, c'est-à-dire nom de conquête. Tel était le 
dessein du western et, d'une manière moins mégalomane, du road movie. La tâche du cinéma contemporain des deux Amériques à l'égard du nom propre Amérique serait celle de faire une sorte de recension des dégâts et des exactions commises au nom de ce nom-ci pendant l'époque projective, tout en le gardant comme le nom d'un rêve inaccompli, d'un amour inassouvi, d'une promesse du bonheur ; Amérique ou América serait le nom des ruines de la fantasmagorie du progrès ${ }^{17}$. C'était déjà le cas pour les films du Nouvel Hollywood (The Last Picture Show de Bogdanovich, par exemple), mais aussi pour une sorte de nouvelle lignée des films aux deux pôles du continent (Zama de Lucrecia Martel, Jauja de Lisandro Alonso, tous les deux des réalisateurs argentins; Meek's Cutoff de l'étasunienne Kelly Reichardt, The Rider de la sino-étasunienne Chloé Zhao).

\section{L'appareillage du territoire}

4 Voilà pour l'exposition de l'état de ma recherche, quelques mois avant le dernier message de Déotte. J'aurais voulu lui dire, et non sans réticence, parce que malgré l'amitié que nous deux entretenions, l'autorité du maître s'imposait encore à moi, que j'avais développé pendant ce temps-ci une intuition fort ambitieuse : je rêvais d'avoir découvert un appareil qui n'était pas sur la liste des appareils modernes que Déotte avait fort bien précisés (la perspective comme appareil matriciel de la modernité, la camera obscura, le musée, la photographie, la psychanalyse, le cinéma ${ }^{18}$ ), mais qui tout de même avait configuré, voire appareillé, notre conception, notre perception et même notre représentation du monde, et cela, comme j'essaierai de le justifier plus loin, de manière littérale. On peut trouver les origines de cet appareil à partir de l'opposition entre peinture et dessin que Déotte développe dans le chapitre faisant suite à son texte sur Lessing dans L'Homme de verre ${ }^{19}$ : en suivant Benjamin ${ }^{20}$, Déotte remarque qu'aux origines, la peinture relevait de la verticalité, elle s'adressait à un spectateur qui doit lever ses yeux; le dessin appartient à l'inscription sur le sol. La peinture se définit par la tache (la couleur) ; le dessin par la ligne (le signe). La peinture est pathétique, c'est-àdire, elle est évènement et donc, elle relève du temps; le dessin, la ligne, est inscription, donc, elle est écriture qui signe un espace. La peinture, la tache, n'a « aucun rapport avec l'arrière-plan ${ }^{21} »$, elle ne se détache pas de celui-ci parce qu'elle devient son propre support, tandis que le dessin a besoin de l'arrière-fond pour s'en détacher, il doit le faire pour devenir visible ou lisible. C'est parce que peinture et dessin se sont soumis à la valeur d'exposition et à l'appareil du musée qu'on s'est habitué à les regarder indifféremment dans une configuration verticale ${ }^{22}$. Benjamin remarque toutefois que le dessin n'est point un tableau : « qu'on regarde en revanche des dessins d'enfants. Presque toujours, le sens interne sera choqué si on les voit verticalement. Il en va de même pour les dessins d'Otto Gross : il faut les coucher à l'horizontale sur une table ${ }^{23}$ ». La peinture entretient un rapport étrange mais déterminant avec le langage par l'intermédiaire du nom : sans le nom, la tache, les formes-figures, ne seraient que des marques ; par-là, la peinture accomplit la mimêsis, puisqu'elle nomme la nature (qui n'a pas de langage). Au contraire, le dessin serait plus proche du nom lui-même : il est un signe qui désigne de l'extérieur un corps, mais aussi, dirais-je, car il est spatial, un territoire. Ce qui est décisif, c'est que le dessin, étant lié à l'horizontale, au sol, est d'emblée un enjeu des signes géométriques, spatiaux; il annonce et implique une cartographie : 
C'est la leçon de Zempléni et Adler ${ }^{24}$ s'agissant des espaces géométriques de la divination archaïque africaine, première cartographie symbolique, magique, d'un territoire, première projection au sol, d'une grille. Donc la projection cartographique sera de l'ordre des signes graphiques et non de la peinture. Parfaite étrangeté de la carte et de la peinture : elle rentre dans le cadre de l'inscription sur l'espace $^{25}$.

C'est ce que montrent encore une fois les dessins d'enfants, ils n'expriment pas d'emblée un évènement pictural, même si les lignes comportent de la couleur : ce sont des labyrinthes, des schémas, des modes d'emploi, des feuilles de route, des territoires magiques et symboliques à parcourir avant d'être expressifs. Ce n'est qu'à partir d'une fantasmagorie des adultes projetée sur les enfants qu'on peut songer qu'un dessin enfantin est pathétique à l'instar de la peinture. Même pour découvrir un crime, comme dans les séries TV américaines, il faut comprendre le dessin enfantin comme une sémiotique de l'espace, de leur territoire. Si le dessin archaïque ou enfantin est question de signes dans l'espace, voire de signes territoriaux, si du moins ces dessins impliquent une proto-cartographie symbolique, magique, il faut peut-être en conclure qu'ils impliquent l'éventualité d'un appareil qui opèrerait par le biais de signes graphiques et de noms qui tracent un territoire : je suggère que cet appareil ne serait rien d'autre que la cartographie moderne.

\section{Le monde comme représentation}

6 Pourquoi alors privilégier la cartographie moderne à toute autre cartographie, alors que l'on pourrait dire qu'il y a une sorte de désir cartographique ou encore une protocartographie dans d'autres sociétés, dans d'autres civilisations et peuples? Les Aztèques, les Mayas, les Mixtèques, les Aborigènes de l'Australie, les peuples autochtones de l'Amérique du Nord, les Chinois, les Indiens, les Japonais, les anciennes civilisations de Çatal Höyük et Nippur, les Grecs et les Romains ont élaboré des cartes assez complexes et efficaces à l'égard des fonctions qu'ils devaient accomplir: navigation, agriculture, élevage, partage, repérage et marquage divin et politique des territoires. Bref, les peuples n'ont attendu ni la triangulation avec laquelle Leon Battista Alberti a dressé un plan de Rome ${ }^{26}$ ni encore la projection perspectiviste avec ses méridiens et parallèles pour faire des cartes. Ce n'est pas, du moins pas essentiellement, la précision technique ou scientifique qui distingue l'appareillage de la cartographie moderne de ces cartes qui, avec les cartes médiévales déploient un protoappareillage cartographique de la Terre et des océans, du land, du territoire. On peut faire l'hypothèse que ce qui va constituer comme appareil la cartographie moderne, européenne à l'origine, c'est sa rencontre avec la représentation. Déjà à l'âge classique, Antoine Arnauld et Pierre Nicole remarquaient que :

Quand on considère un objet en lui-même et dans son propre être, sans porter la vue de l'esprit à ce qu'il peut représenter, l'idée qu'on en a est une idée de chose, comme l'idée de la terre, du soleil; mais quand on ne regarde un certain objet que comme en représentant un autre, l'idée qu'on en a est une idée de signe, et ce premier objet s'appelle signe. C'est ainsi qu'on regarde d'ordinaire les cartes et les tableaux. Ainsi le signe enferme deux idées : l'une de la chose qui représente, l'autre de la chose représentée, et sa nature consiste à exciter la seconde par la première ${ }^{27}$.

Lorsque le signe représentatif devance le symbole, dont la nature est plutôt analogique, ce sont tous les rapports entre la Terre et le territoire, d'un côté, et la cartographie, de l'autre, qui sont bouleversés. Il est possible de dire que c'est par la cartographie 
moderne qu'on entre effectivement dans «L'Âge des conceptions du monde ». En effet, ce que la cartographie moderne a inventé, ce n'est pas tellement la représentation du monde, mais le monde comme représentation. Heidegger a eu une intuition importante : ni les Grecs ni les médiévaux n'auraient pu concevoir le monde comme représentation ou image conçue (Weltbild), car pour les seconds, l'étant est envisagé comme ens creatum, donc toujours en relation à la cause créatrice, tandis que pour les premiers, c'est plutôt l'étant qui regarde l'homme ${ }^{28}$. Ce n'est qu'à l'âge moderne qu'on peut concevoir un "tableau de l'étant dans sa totalité29 ", c'est-à-dire que l'étant dans sa totalité se comprend comme dérivé de sa représentation pour un sujet. Heidegger s'attaque alors à la science et à la technique modernes et la diatribe qu'il entreprend contre Descartes qui, pour Heidegger, est le responsable philosophique de l'assimilation complète de l'étant à la représentation, l'empêche de considérer que la représentation ne se fait monde que par des techniques concrètes. La science ne peut dresser un tableau général de l'étant parce, comme Heidegger lui-même le constate, elle est toujours particulière et telle est l'essence de la science moderne en tant que projet. Tandis que la technique, elle, est concrète, et ne peut donc tracer un tableau de la totalité que si elle abstrait des éléments pour les rendre représentables.

8 On peut suggérer que Heidegger a adultéré la notion de monde, pour la construire comme quelque chose de plus grand que la Nature et le $\operatorname{Cosmos}^{30}$ : pour Heidegger, le monde construit par la modernité ne serait pas ce monde-ci, mais la représentation infinie obéissant à une mathesis universalis que l'on trouve, outre chez Descartes, chez Leibniz, chez Kant, et, d'une autre manière, chez Spinoza. Néanmoins, monde (Welt) est distinct de représentation (Vorstellung) et l'enjeu du texte de Heidegger se trouve dans cette distinction. C'est que la différence entre nature, cosmos et monde n'est pas seulement quantitative. On peut dire qu'elle devient quantitative parce qu'il y a une différence qualitative : pour Heidegger, nature, cosmos et histoire font partie du monde sans qu'ils puissent l'épuiser ${ }^{31}$. Ils ne l'épuisent pas parce que le monde est corrélatif au Dasein et donc, non pas uniquement à l'étant, mais à l'être. Lorsque Heidegger décrit les chaussures du tableau de Van Gogh qu'il analyse dans L'Origine de l'œeuvre d'art comme appartenant à une paysanne (à tort, d'après Meyer Schapiro: les chaussures seraient celles de Van Gogh lui-même ${ }^{32}$ ), il faut comprendre que le monde du Dasein de la paysanne est en quelque sorte inscrit sur ces dernières ${ }^{33}$. Heidegger décrit ce monde rustique : la pénibilité du travail dans le champ, les marques de la terre mouillée sur le cuir des chaussures. Peut-on suggérer que Heidegger se trouve sans doute pris par la fantasmagorie d'un rapport au monde sans médiation technique? Peut-on suggérer encore que même pour décrire ce rapport comme étant au-delà de la technique, c'est-àdire comme relation authentique du Dasein avec l'être, il a dû pactiser avec la technique picturale et avec une description en quelque sorte technique des chaussures? On peut accorder que le rapport du Dasein au monde est distinct de son expression par l'art. En outre, Heidegger n'exclut pas la dimension technique de l'art, bien au contraire: il rappelle qu'art et technique ne se distinguaient pas dans leur origine grecque, ils étaient le même mot. Ce n'est qu'avec la longue et laborieuse chute de l'Occident dans le processus de l'oubli de l'être qu'art et technique se sont trouvés séparés. Dans la dernière étape de ce processus, la technique moderne, liée alors à la représentation, tout en étant un mode de dévoilement, menace le rapport d'écoute de l'être que les Grecs ont été les premiers à établir, mais que malgré tout, l'art serait en mesure de conserver. Pour Heidegger, l'art peut conserver le rapport entre le Dasein et le monde, 
dans l'attente d'un âge où se rétablirait un mode en quelque sorte analogue à celui des origines, où l'art et la technique ne seraient qu'une seule et même chose.

Il s'agit alors de l'établissement d'un rapport d'authenticité entre l'art et l'être. C'est parce que l'art maintient la relation originaire entre le Dasein et le monde qu'on peut dire que l'art, d'après Heidegger, est une technique d'authentification de cette relation. Par conséquent, la fantasmagorie demeure, pour autant qu'on admette que la relation entre expression du monde par le biais des techniques, dont l'art ne serait qu'une forme parmi d'autres, et le monde comme fonds originaire et terrestre des motifs et matériaux, comporte toujours une marge de virtualité, voire d'hallucination. Autrement dit, l'authenticité que Heidegger croit avoir trouvée ne peut se défaire complétement de la fantasmagorie des origines. Mieux, son authenticité n'est que la fantasmagorie de l'origine, la rêverie du Natal: un Allemand $d u x^{e}$ siècle qui pense être l'héritier exclusif des Grecs anciens, par exemple. Dès lors, on peut dire, en suivant Déotte, que tout appareil est toujours investi par un " plasma imaginal », qui conforme les matériaux d'une rêverie collective (Benjamin ${ }^{34}$ ). De même, on peut suggérer, en prolongeant la pensée de Déotte, que ce n'est pas le réel sensible ou encore l'être dans son authenticité, mais les songes et les rêveries de l'humanité (les rêves des cabalistes et chamans, les fables et mythes des peuples, l'obsession du souvenir des bons vieux temps relayée par la photographie) qui établissent à chaque fois les fondations du rapport entre le collectif et la terre, le territoire, le monde. Ce n'est pas l'art comme entité éternelle qui authentifie et rassure la communication de l'homme avec le monde, la nature, la physis ou Dieu, mais les appareils (dont les œuvres d'art dépendent, comme dans le cas du musée) comme configurations qui investissent les rapports entre le collectif et le réel, ce qui déracine et émancipe les relations entre les corps et les lieux. Cela, de telle sorte que la cartographie moderne ne serait qu'un certain aboutissement d'un processus qui aurait commencé dans la phase magique primitive décrite par Simondon : à l'aube de l'humanité, il n'y aurait ni objets ni sujets, mais un univers ou un monde structuré en figure et fond par le biais d'une réticulation des lieux (montagnes, vallées, fleuves) et moments privilégiés (saisons, fêtes, commémorations ${ }^{35}$ ). Le sommet d'une montagne serait la figure ou le point-clé qui surplombe et domine une contrée qui est comme son fond; de même pour un jour férié par rapport auquel l'année prend sens et valeur. Pour Simondon, le premier objet à être apparu serait déjà un objet technique et le premier sujet serait la divinité : technique et religion, voire le domaine qu'on pourrait nommer spiritualité, surgissent de la fragmentation de ce monde comme phases, qui donneront naissance à d'autres phases : la technique se fragmentera à son tour en théorie (la science) et technique pratique et la religion en éthique et dogme ${ }^{36}$. Avant cette deuxième fragmentation, néanmoins, l'on trouve le surgissement de la pensée esthétique au moment du déphasage même de l'univers magique en technique et religion. Pour Simondon, l'esthétique ne serait pas une phase à proprement parler, mais une tendance, une médiation qui comble l'écart entre technique et religion. L'esthétique conserve, sous le mode d'une analogie de portée cosmique, la fonction de totalité de la phase magique primitive. Comme écrit Simondon : «la tendance esthétique est l'œcuménisme de la pensée ${ }^{37}$ ».

On accordera qu'il y a des ressemblances entre la phase magique de Simondon et le rapport originaire du Dasein à l'être chez Heidegger. Reste que la phase magique chez le premier n'est pas un moment dialectique qui serait surpassé par d'autres phases postérieures. En quelque sorte, elle est toujours présente dans les déphasages successifs et n'a de sens qu'en se rapportant à d'autres phases. À cet égard, loin d'être un passé 
réel révolu, dont l'esprit authentique réclame d'être récupéré sous la figure du destin d'un peuple historique comme chez Heidegger, la phase magique devient l'hypothèse toujours agissante d'un fonds immémorial ou d'un passé commun à l'humanité qui se manifeste dans l'esthétique comme besoin de cosmicité. Simondon surpasserait ainsi l'eurocentrisme de Heidegger. Or, dans la mesure où Simondon postule que la phase magique implique déjà une réticulation ${ }^{38}$, on peut concevoir qu'elle renvoie à une proto-cartographie pré-technique et magique, antérieure à la division entre technique et religion. Pourtant, en suivant $L a$ Psychologie historique du carnaval de Florens Christian Rang, un texte cher à Déotte et à Benjamin, on peut esquisser l'hypothèse que cette première réticulation se forme à partir du ciel étoilé, qui conformerait la toute première surface d'inscription. D'après Rang, les astronomes chaldéens (mais on pourrait en dire autant des astronomes mayas) auraient eu l'intuition fondatrice et géniale de reproduire l'ordre et la régularité céleste sur la Terre, en créant ainsi le premier calendrier, mais aussi peut-être la toute première morale, le premier ordre religieux : «Ce que le ciel commande doit advenir sur terre ${ }^{39}$ ». On peut donc prolonger la pensée de Simondon, pour suggérer que le monde de la réticulation magique impliquerait un télescopage, un jeu de miroirs entre la voûte céleste et le territoire. Ce jeu donnerait dans un premier moment toute la force cosmique aux points-clés et aux moments-clés terrestres (alors chargés de la force stabilisatrice, régulière et cyclique des étoiles fixes et des constellations). Cependant, dans un second moment indéterminé, les points et moments-clés seraient sursaturées par l'irrégularité de certains astres (les planètes), créant ainsi l'éventualité d'un instant de chaos légal sur terre : le carnaval, la «légale suspension des lois ${ }^{40}$ ». Les premiers chamanes auraient tracé la première réticulation, la première carte sur le sol en reproduisant et en adaptant l'ordre et les excentricités célestes sur la terre. Il y aurait donc, dès la nuit des temps, une proto-écriture, un proto-appareillage presque cartographique, ou du moins une sorte de besoin, une espèce de délire de la part de l'humanité de tracer les règles de la terre, afin de se donner une place dans le Cosmos, mieux, dans le Chaosmos par le biais d'un nom propre inscrit sur le sol. Il n'y a pas de mythes, de fables, d'histoire et de généalogie, donc pas de territoires, sans noms propres. On peut alors penser que la réticulation magique de Simondon ne peut être conçue que comme un dessin, un croquis, un schéma, une carte sur le sol, qui réclame, qui suppose l'éventualité d'être nommée. La cartographie moderne aurait accompli, sur le mode de la représentation, le désir primitif de l'humanité de se donner un lieu dans le monde et de donner à ce lieu un nom. La contribution de Déotte à une pensée de la phase magique primitive consiste en ceci qu'il a vu que la sursaturation par laquelle Simondon décrivait le déphasage de l'unité magique primitive en technique et en religion, et donc la naissance de l'esthétique comme médiation œcuménique, reste abstraite si l'on ne la justifie pas par l'appareillage. En effet, ce n'est que par les appareils que l'humanité arrive à se détacher d'un fonds qui par lui-même pourrait perdurer sans jamais se déphaser ${ }^{41}$. C'est pourquoi, à la fin de sa vie, Déotte préférait la notion de cosmétique à celle d'esthétique (la première aurait un rapport immanent à l'unité que la seconde n'aurait pas ${ }^{42}$ ). Les appareils, donc, sont cosmétiques dans le sens où ils mettent en place la fonction de totalité, en opérant une médiation entre la concrétisation technique et la spiritualisation de la religion:

Les appareils cosmétiques mettent tous en place de nouvelles réticulations. [Ils] procèdent à des appariements productifs, réduisant l'essentielle hétérogénéité $d u$ monde, non seulement en classant et déclassant, mais en générant des réalités 
nouvelles, des configurations de la singularité quelconque, de l'être-ensemble et de la temporalité, jamais vues auparavant ${ }^{43}$. modernes, on n'aurait pas pu concevoir le monde comme représentation. Pour autant, il ne néglige pas seulement les techniques cartographiques et astronomiques, qui effectivement dressent des tableaux représentatifs de la Terre et du Cosmos connus; Heidegger ne s'aperçoit pas non plus que la sursaturation désirante qu'il nomme «époque des conceptions du monde" (Zeit des Weltbildes) serait peut-être l'effet fantasmagorique du dressage du monde (c'est-à-dire de la Terre devenue Monde et non pas du monde en tant que manifestation de l'être) opéré notamment par la cartographie occidentale. Il faudrait prendre au sérieux le mot d'ordre de Heidegger pour le détourner au profit d'une pensée de l'appareil cartographique moderne : nous essayons sans doute de nous inspirer encore du monde comme représentation. Seulement, il ne s'agit aucunement d'un temps schopenhauerien, il s'agit plutôt d'une époque qui peut-être a touché à sa fin pendant la dernière décennie, avec un monde qui est réglé par des pulsations et les stimuli des réseaux numériques pour lesquels le monde des cartes représentatives n'est pas nécessaire. Il faut pourtant décrire ce monde, qui sans doute est en train de disparaître : dire que le monde est représentation est dire que la représentation y est première. C'est aussi dire que le signe qui se substitue au symbole, se distingue moins pour être autonome que pour commander la réalité et l'être : lors d'un vol en avion, nous ne voyons pas d'en haut les contours de la pointe de la péninsule de la Basse-Californie comme s'il s'agissait d'une terra incognita qui mériterait d'être explorée et cartographiée. Bien au contraire: c'est la représentation de la péninsule dans la carte qui appareille toute notre vision actuelle. Nous nous rappelons que d'un côté, on a l'océan Pacifique et, de l'autre, une mer intérieure: le golfe de Californie, aussi connu comme le Mar de Cortés; et le savant pensera sans doute aux premières cartes de la Californie, où celle-ci apparaissait comme une île.

\section{L'appareillage cartographique}

Il faut relire le célèbre texte De la rigueur de la science de Jorge Luis Borges comme une fable de la représentation: les cartographes s'empressent de construire des cartes de l'Empire de plus en plus précises. À un certain moment, « les Collèges des Cartographes levèrent une Carte de l'Empire, qui avait le Format de l'Empire et qui coïncidait point par point avec lui ${ }^{44} \%$. Les générations ultérieures négligeront la carte et l'abandonneront : «Dans les Déserts de l'Ouest, subsistent des Ruines en lambeaux de la Carte, habitées par des Animaux et des Mendiants. Dans tout le Pays, il n'y a plus d'autres reliquats des Disciplines Géographiques ${ }^{45} »$. Ce n'est pas que le réel ait disparu avec la représentation cartographique moderne, comme a pu l'affirmer Jean Baudrillard dans sa lecture de ce texte ${ }^{46}$, parce qu'il n'y aurait point de réel pur, hors des appareillages et hors des techniques par lesquels les choses et les rapports avec la terre sont compris : l'humanité n'a pas attendu la représentation pour faire des simulacra et pour faire disparaître, donc, avant la lettre, le réel baudrillardien. Il ne s'agit pas non plus de constater que la carte n'est pas le territoire : «A map is not the territory ${ }^{47}$ ", postulait Alfred Korzybski. En effet, il est évident que le territoire n'est pas sa représentation. Celle-ci implique tout autre chose : l'humour du jeu de la grandeur démesurée des cartes de Borges ne peut être saisi que si l'on prend en compte le fait

Appareil, 22 | 2020 
que la précision de la représentation se perd à grande échelle. La représentation doit être précise, sans imiter ou sans s'apparenter à la chose. C'est la chose qui se construit et se comprend à partir des paramètres de la représentation, du disegno cartographique, de la ligne graphique et du nom. Voilà pourquoi le texte de Borges provoque le rire avant même de finir : ni les cartographes de l'Empire, ni Baudrillard n'ont compris que le texte joue avec l'effet de la représentation. L'effet de réalité des cartes n'est que le résultat fantasmagorique de l'appareillage cartographique : le globe et la Terre ne sont pas la carte, mais on ne peut les reconnaître sans la mise en scène de la représentation. Sans la cartographie moderne, la Basse-Californie ne serait qu'une frange de terre parmi d'autres, un morceau de terre entouré d'eau.

Invention prodigieuse qui aurait fait du monde moderne concret, c'est-à-dire, de la terre comme globe, l'effet de sa représentation sur des cartes, notamment sur des planisphères. Ce n'est qu'à ce moment-là que la géopolitique naît à proprement parler : des dirigeants d'empires, des hommes politiques et des économistes, des savants mais aussi des colons, des aventuriers et de simples migrants, et encore des gens qui ne quitteront jamais leur coin du monde s'empresseront pour accoupler leurs désirs au monde représenté. On ne peut pas affirmer l'inverse, dans la mesure où si puissants que soient les efforts pour adapter le monde représenté aux désirs, on ne peut pas changer la configuration et la grandeur des territoires: on ne peut que les masquer, dans certaines limites, en exagérant l'importance d'une contrée, par exemple. L'Europe sera toujours un appendice de l'Asie en termes géographiques et cartographiques. On a pu toutefois essayer de prolonger sa sphère d'influence: par la colonisation, par le peuplement, par la religion, par la culture, par la guerre, mais toujours dans un monde donné comme représentation par les cartes. On dira alors qu'on essaie de faire passer un dispositif de pouvoir pour un appareil. Déotte insistera sur ce point : un appareil n'est pas un dispositif, parce que ce dernier n'offre ni innervation ni temporalité commune, collective. Au contraire de l'appareil, qui absorbe l'évènement en mettant en rapport les corps avec leur autre, qui est la loi pour fonder une temporalité immanente, inattendue, non programmée à l'avance, le dispositif dispose les corps, les choses, pour une fonction ou un programme dont on pourrait dire qu'il lui est externe (le savoir, le pouvoir, le temps du capital ${ }^{48}$ ). Il serait commode de conclure que la cartographie moderne n'a eu pour fonction principale que de soumettre les corps de la terre aux besoins du pouvoir, notamment occidental, aux revendications des États, des militaires, des élites qui gèrent le capital. Telle serait la position de John Brian Harley qui, dans une inspiration foucaldienne, conclut que sous une apparence de scientificité et d'objectivité, la cartographie occidentale n'est qu'une construction sociale soumise aux pouvoirs temporels ${ }^{49}$. Les cartes ne seraient donc que le dispositif idéologique de la modernité colonisatrice, impérialiste, voire fasciste. Cette conclusion ne revient-elle pas toutefois à oublier que la photographie, le cinéma et la perspective ont aussi été utilisés comme des dispositifs de contrôle des individus et des collectifs, au point parfois de devenir les rouages d'une machinerie de tuerie massive? Reste que les appareils ne s'arrachent jamais définitivement aux enjeux de pouvoir mais, au contraire des dispositifs, ils sont capables de rompre avec les fantasmagories en montrant par des œuvres inédites ce qui se joue dans chaque époqualité ${ }^{50}$. Pour Déotte, ce sont donc les œuvres qui mènent l'appareil vers un seuil où le programme idéologique ou de pouvoir ne peut plus tenir et s'effondre en quelque sorte. Ce serait le cas du cinéma d'Eisenstein, qui aurait rompu avec la fantasmagorie de la vedette 
(Starkultus ${ }^{51}$ ) énoncée par Benjamin. Cependant, qu'en est-il de la cartographie moderne? A-t-elle besoin d'œuvres pour s'arracher au programme?

\section{Le temps de la nomination}

Dans un premier moment, on peut dire que la cartographie moderne ne peut pas être réduite à un pur enjeu ou dispositif de pouvoir, parce qu'elle a toujours accompli des fonctions productrices qui ont apporté des bénéfices à l'humanité: agricoles, commerciaux, économiques, industriels, sociaux, politiques. Il faut tout de même ajouter également, dans un deuxième moment, qu'elle ne saurait se réduire à un objet technique qui accomplirait des fonctions diverses (productrices ou destructives) comme un moteur, un circuit électrique ou un processeur électronique. Certes, on peut toujours utiliser une carte pour envisager des territoires à conquérir ou des objectifs militaires à anéantir. Malgré cela, ici encore, il en va de même que pour la photogrammétrie : si la cartographie est un appareil, elle peut toujours être mise au service des desseins des pouvoirs, tout comme l'appareil photographique. Mon hypothèse est que l'enjeu de la cartographie moderne comme appareil se trouve dans un devenir qui va de son émergence comme objet somptuaire, jusqu'à son internalisation collective où ses aspects techniques tendent à s'effacer pour apparaître aux yeux de la collectivité comme un constat du réel. Même avant la cartographie moderne, les cartes avaient des fonctions qui ne se réduisaient pas à un usage technique immédiat : il s'agissait aussi pour les élites d'y voir un déploiement du monde où elles jouaient un rôle narcissique. On a dressé des cartes à la mesure de la grandeur du commandeur, roi ou empereur. Avoir des cartes aux murs de sa demeure a toujours été une question de prestige, plus que de pouvoir. La cartographie moderne naît quand la technique de triangulation, déjà mise en œuvre par Alberti, mais aussi par des cartographes qui ont tracé des portulans, rencontre la projection issue de la perspective. Cela était nécessaire, parce qu'il fallait rendre compte de la courbure et de la tridimensionnalité de la Terre sur le papier à deux dimensions. Plus l'échelle de la carte est grande, plus la distorsion est importante. La projection est alors une manière de contrôler la distorsion. Le célèbre planisphère Mercator distord les échelles des continents non pas pour répondre au besoin de faire apparaître les terres du Nord, dont la petite Europe, plus grandes par rapport à d'énormes territoires comme le Brésil, mais parce que c'est la seule projection du monde où l'on peut tracer sur la carte des trajets en lignes droites, sans qu'on ait besoin de tracer des trajets courbes ${ }^{52}$. Malgré les réserves que Déotte a toujours eues à l'endroit de l'imprimerie face à la perspective ${ }^{53}$, c'est par l'imprimerie que les cartes modernes vont se populariser. Elles ne pourront plus être réduites à des objets somptuaires destinés à faire valoir le prestige des puissants, ni encore à des objets utiles pour la production ou la destruction. Ce sont les atlas et les cartes dessinés depuis la fin du Moyen Âge qui auront joué le rôle d'œuvres pour l'appareil cartographique: la Tabula Rogeriana de Muhammad Al-Idrisi, l'Atlas portulan de Battista Agnese, le Theatrum Orbis Terrarum d'Abraham Ortelius, la carte de Tenochtitlán que Cortés envoya à l'empereur Charles V, l'Atlas Maior de Joan Blaeu, les Cuvres nautiques de Jacques Devaulx; puis les instituts cartographiques comme Justus Perthes en Allemagne, Rand McNally et la National Geographic aux États-Unis. Or, on peut esquisser l'hypothèse que ces cartes (où la représentation, c'est-à-dire, le commandement du signe qui absorbe son référent dans la ligne graphique et le nom, passe désormais par la projection) auront pour principaux destinataires des musées, 
des établissements scolaires et des bibliothèques, plus que des foyers bourgeois ou des casernes militaires. Donc, la cartographie moderne serait un cas d'appareil introjecté dans le plasma imaginal du collectif par la scolarisation massive, d'abord en Occident et puis à peu près partout dans le monde. Même dans les pays et les contrées les plus indigentes, il est possible de trouver, collés côte à côte au mur, une carte de l'endroit où l'on se trouve et un planisphère.

« Mais vous faites l'éloge de l'endoctrinement idéologique de la part de l'État ! » diront les bonnes consciences. Je crois pourtant que c'est une pauvre lecture de croire que l'humanité a été toujours attachée aux mots d'ordre scolaires. Ce qui, dans les cartes, va plus loin que les mots d'ordre, c'est l'absorption collective et donc, l'appropriation massive des surfaces, des figures, des contours, des contrées, des rivages, des villages, des quartiers et des rues, des lisières et des frontières, des magnitudes, même des textures : un Mexicain reconnaît les contours de son pays, toute comme un Français, un Malaisien, une Alsacienne ou une Togolaise : on fera des stickers, des dessins, des logos et des drapeaux pour soutenir toutes sortes de revendications. Un Chilien verra que la figure de son pays est parmi les plus étranges du monde; tandis que les Mapuches du sud du Chili et de l'Argentine verront dans la même carte l'occasion de réclamer leur territoire ancestral, tout comme les Kurdes, qui revendiquent un État propre. Riposter en affirmant que les frontières sont artificielles et rappeler combien de maux elles ont suscité, c'est, dans ce cas, se tromper d'adversaire: les hommes tracent toujours des limites, même si c'est pour les dépasser impunément par la suite. L'époque de la représentation n'implique aucun désenchantement du monde. Bien au contraire, c'est par les cartes modernes que l'ancienne réticulation magique en points-clés s'actualise et que l'ancien besoin de se donner une place, un chez soi dans le monde, a littéralement lieu.

Ce sont les cartes projectives qui ont créé l'appareillage moderne du monde. Ce sont elles qui ont créé l'évènement même du monde et auront peut-être convié les hommes à devenir des contemporains les uns des autres dans la représentation. Reconnaître la silhouette d'un pays ou d'une petite contrée, les méandres de la rivière de notre enfance ou le large de la côte qu'on n'a jamais visitée, c'est déjà une manière de se situer parmi les gens appartenant à la même filiation et devant autrui. En ce sens, toute carte est une carte politique. Si la cartographie projective peut être située à côté des autres appareils modernes, c'est parce qu'elle a une temporalité propre. On dira qu'en principe une carte n'est pas de l'ordre du temps, parce qu'à l'instar du dessin et au contraire de la peinture chez Benjamin et Déotte, le nom et la ligne graphique ne relèvent pas de la pathétique propre à l'évènement. Au contraire, le nom et la ligne graphique entretiennent un rapport spatial avec la surface ${ }^{54}$. On suggère pourtant que la temporalité projective serait celle de la nomination : à l'instar de photographies qui réclament d'être nommées, d'après Benjamin ${ }^{55}$, tout territoire, toute surface sur la terre réclame un nom. Le temps de la nomination cartographique serait celui du recommencement, temps indéterminé qui comblerait pourtant l'écart entre l'instant et l'éternité par le don. Parce que tout nom propre est un don, il est aussi la marque d'un destin. Y aurait-il une rêverie collective plus puissante et plus archaïque que celle consistant à investir la terre en la nommant? Nommer les choses, les plantes, les animaux, les hommes, la terre et les mers, est une prérogative divine et enfantine à la fois. Tout enfant qui lit pour la première fois une carte, tout adulte qui la relit avec soin, invente le sens des noms de la carte. De la même manière que les noms n'ont pas 
de signification, ils n'ont pas non plus de sens préalable, ils sont des dons qui investissent les surfaces cartographiques.

Il se peut que nous soyons en train de sortir de cette époque, puisque la surface d'inscription numérique n'a plus besoin de cartes qui durent, vieillissent et deviennent anachroniques par la suite : la cartographie numérique est celle du temps réel où les cartes sont des logiciels qui changent, se métamorphosent et s'améliorent sans cesse. On n'est pas en mesure de dire que le monde de la cartographie numérique ne comporte pas un monde sous une nouvelle configuration. Ce que j'ose dire, c'est que ce monde n'appartiendra plus à la représentation. Dans ce monde nouveau, pourtant, resteront encore des ruines de la cartographie moderne, dans le désert de Google Maps. Ce que je voulais dire à Jean-Louis Déotte est que je travaille fort sur ce chantier ruineux et que ma thèse à ce sujet est qu'il n'y aurait pas eu de cosmétique moderne sans cartes et qu'il se peut que nous ayons été contemporains du monde tel qu'il a été représenté par les cartes projectives. Cela, on le sait déjà : tout comme mon message pour Déotte, la philosophie arrive toujours trop tard.

\section{Postface}

À l'automne 2012, j'ai aidé Déotte à défricher une partie de son jardin à Paris. Vers la fin de la journée, il m'a offert un petit et étrange cadeau en retour : une sorte de grand caillou gris, lourd et un peu poreux en forme de brique. Il m'a dit qu'il s'agissait d'un pavé qu'il avait ramassé près de la rue Gay-Lussac dans le Quartier latin en mai 1968. Suite à ce cadeau, j'aurais aimé lui poser cette question: y a-t-il un appareil pour l'amitié ? Est-ce que la philosophie n'aurait pas été, du moins pour les Grecs et peutêtre pour nous deux aussi, rien d'autre qu'un appareil, décadent et merveilleux à la fois, pour entretenir l'amitié sous le prétexte de la sagesse, toujours inatteignable?

\section{BIBLIOGRAPHIE}

Adler Alfred et Zempléni Andras, Le Bâton de l'aveugle. Divination, maladie et pouvoir chez les Moundang du Tchad, Paris, Hermann, coll. « Savoir », 1972.

Alberti Leon Battista et Raphaël, Descriptio urbis Romae ou comment faire le portrait de Rome [1444], introd. et trad. du latin B. Queysanne, Paris, Éditions de la Villette, coll. « École d'architecture de Grenoble », 2002.

Arnauld Antoine et Nicole Pierre, La logique ou L'art de penser [La logique de Port-Royal ; 1662], Paris, Gallimard, coll. « Tel », 1992.

Baudrillard Jean, Simulacres et simulation, Paris, Galilée, 1981, coll. « Débats ».

Benjamin Walter, « Das Kunstwerk im Zeitalter seiner technischen Reproduzierbarkeit », in Gesammelte Schriften, première rédaction (Erste Fassung) [1935], t. I-2, p. 431-470, deuxième rédaction (Zweite Fassung) [1936], t. VII-I, p. 350-384, troisième rédaction (Dritte Fassung) [1939], t. I-2, p. 471-508, Francfort-sur-le-Main, Suhrkamp, 1991. 
Benjamin Walter, «L'œuvre d'art à l'époque de sa reproduction mécanisée » [1936], trad. de l'allemand P. Klossowski, in Gesammelte Schriften, t. I-2, Francfort-sur-le-Main, Suhrkamp, p. 709-739.

Benjamin Walter, « Nouveaux paralipomènes à l'œuvre d'art à l'époque de sa reproductibilité technique (B. Crise de la peinture. Peinture et dessin) » [1936], in Écrits français, introd. et notes J.M. Monnoyer, Paris, Gallimard, coll. « Bibliothèque des idées », 1991, p. 190-192.

Benjamin Walter, « Peinture et graphisme. De la peinture ou le signe et la marque » [1917], trad. de l'allemand P. Pénisson et présenté par Y.-A. Bois, La part de l'œil, nº 6, 1990, p. 10-15.

Benjamin Walter, « Petite histoire de la photographie » [1931], trad. de l'allemand M. de Gandillac, revue par P. Rusch, in Euvres, t. II, Paris, Gallimard, coll. « Folio. Essais », 2000, p. 295-321.

Benjamin Walter, « Sur la peinture ou : signe et tache » [1917], trad. de l'allemand P. Rusch, in Euvres, t. I, Paris, Gallimard, coll. « Folio. Essais », 2000, p. 172-178.

Benjamin Walter, « Über Sprache überhaupt und über die Sprache des Menschen » [1916], in Gesammelte Schriften, t. II-1 Aufsätze Essays Vorträge, Francfort-sur-le-Main, Suhrkamp, 1991, p. 140-157 [ « Sur le langage en général et sur le langage humain », trad. de l'allemand M. de Gandillac revue par R. Rochlitz, in Euvres, t. I, Paris, Gallimard, coll. « Folio. Essais », 2000, p. 142-165].

Borges Jorge Luis, « De la rigueur de la science » [1946], in L'Auteur, Euvres complètes, t. II, trad. de l'espagnol J.-P. Bernès, R. Caillois, C. Esteban et al., Paris, Gallimard, Bibliothèque de la Pléiade, 2010 , p. 57.

Déotte Jean-Louis, « Benjamin, le corps de la couleur », in L'homme de verre. Esthétiques benjaminiennes, Paris, L’Harmattan, coll. « Esthétiques », 1998, p. 99-123.

Déotte Jean-Louis, Cosmétiques. Simondon, Panofsky, Lyotard, La Plaine Saint-Denis, Éditions des maisons des sciences de l'homme associées, 2018.

Déotte Jean-Louis, L'Époque des appareils, Paris, Lignes et Manifestes, 2004.

Déotte Jean-Louis, « Lessing, le corps, le nom », in L'homme de verre. Esthétiques benjaminiennes, Paris, L'Harmattan, coll. « Esthétiques », 1998, p. 79-98.

Déotte Jean-Louis, Oubliez! Les ruines, l'Europe, le musée, Paris, L'Harmattan, coll. « La philosophie en commun », 1994.

Déotte Jean-Louis, Qu'est-ce qu'un appareil ? Benjamin, Lyotard, Rancière, Paris, L'Harmattan, coll. « Esthétiques », 2007.

Déotte Jean-Louis, « Un roi pour Israël ?», Appareil, Articles, 2008. URL : http:// journals.openedition.org/appareil/357 [consulté le 27/03/2019].

Domínguez Jiménez Román, « Mito, atmósfera, territorio: una hipótesis cinematográfica del nombre América », L'Atalante. Revista de estudios cinematográficos, n 27, 2019, p. 187-200.

Domínguez Jiménez Román, « Jauja and Meek's Cutoff: the parallel-American ways of rethinking Gilbert Simondon's role of aesthetics in the configuration of humanity » (inédit, publié prochainement dans un recueil de philosophie et cinéma dirigé par Christine Reeh-Peters et Stephan Schmidt chez Brill/Rodopi).

Harley John Brian, The New Nature of Maps. Essays in The History of Cartography, Baltimore, Johns Hopkins University Press, 2001. 
Heidegger Martin, «L’Âge des conceptions du monde » [1949], in Chemins qui ne mènent nulle part, trad. de l'allemand W. Brokmeier, Paris, Gallimard, coll. « Tel », 1986, p. 99-146.

Heidegger Martin, « L'Origine de l'œuvre d'art » [1949], in Chemins qui ne mènent nulle part, trad. de l'allemand W. Brokmeier, Paris, Gallimard, coll. « Tel », 1986, p. 13-98.

Korzybski Alfred, Science and Sanity: An Introduction to Non-Aristotelian Systems and General Semantics [1933], Brooklyn, Institute of General Semantics, 1994.

Kripke Saul, Naming and Necessity, Oxford, Blackwell's, 1981 [La logique des noms propres, trad. de l'américain P. Jacob et F. Recanati, Paris, Minuit, coll. « Propositions », 1982].

Lessing Gotthold Ephraim, Laokoön oder Über die Grenzen der Malerei und Poesie [1766], Stuttgart, Reclam, coll. « Universal-Bibliothek », 1964.

Lyotard Jean-François, Le différend, Paris, Minuit, coll. « Critique », 1983.

Rang Florens Christian, Psychologie historique du carnaval [1909], trad. de l'allemand et notes

F. Rey, Toulouse, Éditions Ombres, 1990.

Rojas Mix Miguel, Los cien nombres de América. Eso que descubrió Colón [1991], Santiago de Chile, Pehuén, 2018.

Schapiro Meyer, "The still life as a personal object - a note on Heidegger and Van Gogh ", in Marianne Leonore Simmel (dir.). The Reach of The Mind: Essays in Memory of Kurt Goldstein, 1998, New York, Springer, 1968, p. 203-209.

Simondon Gilbert, Du mode d'existence des objets techniques, Paris, Aubier-Montaigne, 1958.

Wood Denis, Kaiser Ward et Abramms Bob, Seeing Through Maps. Many Ways to See the World, Amherst, ODT Inc, 2006.

\section{Filmographie}

Alonso Lisandro, Jauja, Buenos Aires, 4L, 2014.

Bogdanovich Peter, The Last Picture Show, Hollywood, BBS Productions, 1971.

Martel Lucrecia, Zama, Rio de Janeiro, Bananeira Filmes, 2017.

Reichardt, Kelly, Meek's Cutoff, New York, Evenstar Films, 2010.

Zhao Chloé, The Rider, Los Angeles, Caviar et Highwayman Films, 2017.

\section{NOTES}

1. Voir Jean-Louis Déotte, «Lessing, le corps, le nom», in L'homme de verre. Esthétiques benjaminiennes, Paris, L'Harmattan, coll. «Esthétiques», 1998, p.79-98. Voir également Gotthold Ephraim Lessing, Laokoön oder Über die Grenzen der Malerei und Poesie [1766], Stuttgart, Reclam, coll. « Universal-Bibliothek », 1964.

2. Voir Walter Benjamin, "Über Sprache überhaupt und über die Sprache des Menschen» [1916], in Gesammelte Schriften, t. II-1 Aufsätze Essays Vorträge, Francfort-sur-le-Main, Suhrkamp, 1991, p. 140-157 ["Sur le langage en général et sur le langage humain », trad. de l'allemand M. de Gandillac, revue par R. Rochlitz, in Euvres, t. I, Paris, Gallimard, coll. « Folio. Essais », 2000, p. 142-165]. 
3. Jean-Louis Déotte, «Un roi pour Israël?», Appareil, Articles, 2008. URL: http:// journals.openedition.org/appareil/357 [consulté le 27/03/2019]

4. Jean-Louis Déotte, Cosmétiques. Simondon, Panofsky, Lyotard, La Plaine Saint-Denis, Éditions des maisons des sciences de l'homme associées, 2018.

5. Jean-Louis Déotte, «Lessing, le corps, le nom », op. cit., p. 94.

6. Ibid., p. 95.

7. Ibid., p. 89.

8. Ibid., p. 92.

9. Ibid., p. 95.

10. Id.

11. Saul Kripke, Naming and Necessity, Naming and Necessity, Oxford, Blackwell's, 1981 [La logique des noms propres, trad. de l'américain P. Jacob et F. Recanati, Paris, Minuit, coll. « Propositions ", 1982].

12. Jean-François Lyotard, Le différend, Paris, Minuit, coll. « Critique », 1983, p. 60.

13. Voir Miguel Rojas Mix, Los cien nombres de América. Eso que descubrió Colón, Santiago de Chile, Pehuén, 2018.

14. Voir Román Domínguez Jiménez, «Mito, atmósfera, territorio: una hipótesis cinematográfica del nombre América ", L'Atalante. Revista de estudios cinematográficos, n² 27, 2019 ; et aussi «Jauja and Meek's Cutoff: The Parallel-American Ways of Rethinking Gilbert Simondon's Role of Aesthetics in the Configuration of Humanity " (inédit, à publier prochainement dans un recueil sur philosophie et cinéma dirigé par Christine Reeh-Peters et Stephan Schmidt chez Brill/ Rodopi).

15. Jean-Louis Déotte, L'Époque des appareils, Paris, Lignes et Manifestes, 2004, p. 248, 316.

16. Il est possible de préciser le nom Amérique comme le porteur d'un effet fantasmagorique lié non seulement à l'écriture projective, mais aussi à l'invention du disegno. Après avoir écrit les deux textes cités plus haut, j'ai trouvé ce passage de Déotte, qui caractérise fort bien le changement de temporalité qui suppose le disegno et la notion de progrès: "Cette capacité de tracer sur un support idéalisé comme transparent ce qui se projette de là-bas, devant la fenêtre du plan de projection, et avance vers le spectateur pour laisser son empreinte, est la clef du dessin entendu par les traités renaissants comme disegno. [...]. C'est l'ensemble de la civilisation occidentale qui va être bouleversé [...] : la monnaie comme moyen d'échange [...] va devenir crédit, c'est-à-dire achat du temps lui-même. Le capitalisme ne suppose pas seulement une ascèse mondaine où la réussite d'un travail est la preuve de l'élection divine [...], mais plus fondamentalement un ethos où le bien-être de tous et de chacun réside dans la capacité de se projeter dans le futur. [...]. La temporalité selon les Lumières était orientée selon un principe qualitatif: le progrès supposant un accomplissement de l'idéalité, la temporalité postmoderne, démocrate-capitaliste, ne connaît que l'accroissement quantitatif: le développement sans limites. [...] A contrario, la temporalité des religions révélées est cyclique ». Jean-Louis Déotte, Cosmétiques, op. cit., p. 7.

17. Il est possible de dire que la question du nom propre Amérique, telle que j'essaie de la développer, comme le nom d'une ruine projective, rejoint sur un autre mode celle de Déotte, pour qui la question de l'Europe est liée indissociablement à la question de sa ruine, de ses ruines. Voir Jean-Louis Déotte, Oubliez! Les ruines, l'Europe, le musée, Paris, L'Harmattan, coll. «La philosophie en commun », 1994, p. 10 et sq.

18. Jean-Louis Déotte, L'Époque des appareils, op. cit., p. 273-297.

19. Jean-Louis Déotte, «Benjamin, le corps de la couleur", in L'homme de verre. Esthétiques benjaminiennes, Paris, L'Harmattan, coll. « Esthétiques », 1998, p. 99-123. 
20. Voir Walter Benjamin, «Nouveaux paralipomènes à l'œuvre d'art à l'époque de sa reproductibilité technique (B. Crise de la peinture. Peinture et dessin) » [1936], in Écrits français, introd. et notes J.-M. Monnoyer, Paris, Gallimard, coll. «Bibliothèque des idées », 1991, p. 190-192. Voir aussi Walter Benjamin, «Sur la peinture ou : signe et tache » [1917], trad. de l'allemand P. Rusch, in CEuvres, t. I, Paris, Gallimard, coll. « Folio. Essais », 2000, p. 172-178.

21. Jean-Louis Déotte, « Benjamin, le corps de la couleur », op. cit., p. 110.

22. Ibid., p. 109.

23. Walter Benjamin, «Peinture et graphisme. De la peinture ou le signe et la marque » [1917], trad. de l'allemand P. Pénisson et présentation Y.-A. Bois, La part de l'œil, nº 6, 1990, p. 13.

24. Je précise : Alfred Adler et Andras Zempléni, Le Bâton de l'aveugle. Divination, maladie et pouvoir chez les Moundang du Tchad, Paris, Hermann, coll. « Savoir », 1972.

25. Jean-Louis Déotte, «Benjamin, le corps de la couleur », op. cit., p. 107.

26. Déotte décrit, à partir de l'ouvrage de Leon Battista Alberti et Raphaël, Descriptio urbis Romae ou comment faire le portrait de Rome, le dressage de ce plan par Alberti comme exemple archaïque d'une "Véritable numérisation non-projective, obtenue par triangulation » dans Cosmétiques, p. 28. De ce fait, Déotte montre que la projection est travaillée par la numérisation depuis le début.

27. Antoine Arnauld et Pierre Nicole, La logique ou L'art de penser [La logique de Port-Royal] [1662], Paris, Gallimard, coll. « Tel », 1992, ch. IV, p. 46.

28. Voir Martin Heidegger, "L'Âge des conceptions du monde", in Chemins qui ne mènent nulle part, Paris, Gallimard, coll. « Tel », 1986, p. 118-119.

29. Ibid., p. 116.

30. Id.

31. Id.

32. Voir Meyer Schapiro, "The still life as a personal object - a note on Heidegger and Van Gogh ", in Marianne Leonore Simmel (dir.). The Reach of The Mind: Essays in Memory of Kurt Goldstein, 1998, New York, Springer, 1968, p. 203-209.

33. Martin Heidegger, "L'Origine de l'œuvre d'art ", in Chemins qui ne mènent nulle part, Paris, Gallimard, coll. « Tel », 1986, p. 34.

34. Voir Jean-Louis Déotte, L'Époque des appareils, op. cit., p. 50-51.

35. Voir Gilbert Simondon, Du mode d'existence des objets techniques, Paris, Aubier-Montaigne, 1958, p. 159-170.

36. Ibid., p. 158.

37. Ibid., p. 181.

38. Ibid., p. 164-165.

39. Florens Christian Rang, Psychologie historique du carnaval [1909], Toulouse, Éditions Ombres, 1990 , p. 27.

40. Id.

41. Voir Jean-Louis Déotte, Cosmétiques, op. cit., p. 18.

42. Id.

43. Ibid., p. 21, 22.

44. Jorge Luis Borges, "De la rigueur de la science ", in L'Auteur, Euvres complètes, t. II, Paris, Gallimard, Bibliothèque de la Pléiade, 2010, p. 57.

45. Id.

46. Voir Jean Baudrillard, Simulacres et simulation, Paris, Galilée, 1981, coll. « Débats », p. 9-10.

47. Alfred Korzybski, Science and Sanity: An Introduction to Non-Aristotelian Systems and General Semantics [1933], Brooklyn, Institute of General Semantics, 1994 p. 58.

48. Voir Jean-Louis Déotte, Qu'est-ce qu'un appareil? Benjamin, Lyotard, Rancière, Paris, L'Harmattan, coll. « Esthétiques », 2007, p. 110-111, 135. 
49. Voir John Brian Harley, The New Nature of Maps. Essays in The History of Cartography, Baltimore, Johns Hopkins University Press, 2001, p. 45-46.

50. Jean-Louis Déotte, L'Époque des appareils, op. cit., p. 106-107.

51. Sur le Starkultus ou « culte de la vedette» (dans la version traduite par Pierre Klossowki) dans L'œuvre d'art à l'époque de sa reproduction technique, voir Walter Benjamin, «Das Kunstwerk im Zeitalter seiner technischen Reproduzierbarkeit ", in Gesammelte Schriften, première rédaction (Erste Fassung) [1935], t. I-2, p. 452, deuxième rédaction (Zweite Fassung) [1936], t. VII-I, p. 370, troisième rédaction (Dritte Fassung) [1939], t. I-2, p. 494, Francfort-sur-le-Main, Suhrkamp, 1991 ; voir également «L'œuvre d'art à l'époque de sa reproduction mécanisée » [1936], trad. de l'allemand P. Klossowski, in Gesammelte Schriften, t. I-2, Francfort-sur-le-Main, Suhrkamp, p. 727.

52. Voir Denis Wood, Ward Kaiser et Bob Abramms, Seeing Through Maps. Many Ways to See the World, Amherst, ODT Inc, p. 8-9.

53. Voir Jean-Louis Déotte, L'Époque des appareils, op. cit., p. 55.

54. Voir Jean-Louis Déotte, « Benjamin, le corps de la couleur », op. cit., p. 107-108.

55. Voir Walter Benjamin, «Petite histoire de la photographie» [1931], trad. de l'allemand M. de Gandillac, revue par P. Rusch, in Euvres, t. II, Paris, Gallimard, coll. « Folio. Essais », 2000, p. 295-321.

\section{RÉSUMÉS}

Bien que l'œuvre de Jean-Louis Déotte dessine une ligne à peu près chronologique des appareils, des normes d'écriture et des cosmétiques tout au long du devenir de la culture et de la technique, on sait que ses analyses les plus précises se centrent sur lesdits appareils modernes, tantôt constituants, tantôt issus de l'écriture projective: perspective, camera obscura, photographie, musée, passages urbains, psychanalyse, cinéma. Le dessein du texte qui suit est de suggérer, du moins comme hypothèse, que l'écriture projective aurait érigé un autre appareil encore: la cartographie moderne. Pour ce faire, il faut évidemment montrer comment la cartographie ne se réduit pas à un dispositif de pouvoir : elle serait aussi un des modes de la rêverie collective qui, dans la modernité, se développe comme un désir de territoire et, plus précisément, comme un désir de monde qui passe par sa représentation projective. Cela implique inévitablement une discussion avec Heidegger, mais aussi une recherche sur l'un des thèmes récurrents chez Déotte : la question du nom propre et de la nomination. C'est parce que la cartographie moderne établit un lien entre les noms propres, la ligne graphique et la surface de représentation, qu'elle arrive à constituer une cosmétique qui évoque l'unité magique primitive de Simondon.

\section{INDEX}

Mots-clés : nom propre, carte, projection, représentation, Amérique 


\section{AUTEURS}

\section{ROMÁN DOMÍNGUEZ JIMÉNEZ}

Profesor asistente à l'Instituto de Estética, Facultad de Filosofía, de la Pontificia Universidad

Católica de Chile ; rdominguezj@uc.cl 